\title{
UCB System Description for the WMT 2007 Shared Task
}

\author{
Preslav Nakov \\ EECS, CS division \\ University of California at Berkeley \\ Berkeley, CA 94720 \\ nakovecs.berkeley.edu
}

\author{
Marti Hearst \\ School of Information \\ University of California at Berkeley \\ Berkeley, CA 94720 \\ hearstaischool.berkeley.edu
}

\begin{abstract}
For the WMT 2007 shared task, the UC Berkeley team employed three techniques of interest. First, we used monolingual syntactic paraphrases to provide syntactic variety to the source training set sentences. Second, we trained two language models: a small in-domain model and a large out-ofdomain model. Finally, we made use of results from prior research that shows that cognate pairs can improve word alignments. We contributed runs translating English to Spanish, French, and German using various combinations of these techniques.
\end{abstract}

\section{Introduction}

Modern Statistical Machine Translation (SMT) systems are trained on aligned sentences of bilingual corpora, typically from one domain. When tested on text from that same domain, such systems demonstrate state-of-the art performance; however, on out-of-domain text the results can get significantly worse. For example, on the WMT 2006 Shared Task evaluation, the French to English translation BLEU scores dropped from about 30 to about 20 for nearly all systems, when tested on News Commentary rather than Europarl (Koehn and Monz, 2006).

Therefore, this year the shared task organizers have provided $1 \mathrm{M}$ words of bilingual News Commentary training data in addition to the Europarl data (about 30M words), thus challenging the participants to experiment with domain adaptation.

Below we describe our domain adaptation experiments, trying to achieve better results on the News
Commentary data. In addition to training on both data sets, we make use of monolingual syntactic paraphrases of the English side of the data.

\section{Monolingual Syntactic Paraphrasing}

In many cases, the testing text contains "phrases" that are equivalent, but syntactically different from the phrases learned on training, and the potential for a high-quality translation is missed. We address this problem by using nearly equivalent syntactic paraphrases of the original sentences. Each paraphrased sentence is paired with the foreign translation that is associated with the original sentence in the training data. This augmented training corpus can then be used to train an SMT system. Alternatively, we can paraphrase the test sentences making them closer to the target language syntax.

Given an English sentence, we parse it with the Stanford parser (Klein and Manning, 2003) and then generate paraphrases using the following syntactic transformations:

1. [NP $\left.\mathbf{N P}_{1} \mathbf{P} \mathbf{N P}_{2}\right] \Rightarrow\left[\mathbf{N P} \mathbf{N P}_{2} \mathbf{N P}_{1}\right.$. inequality in income $\Rightarrow$ income inequality.

2. [NP $\mathbf{N P} \mathbf{P}_{1}$ of $\left.\mathbf{N P}_{2}\right] \Rightarrow$ [NP $\mathbf{N P}_{2}$ poss $\left.\mathbf{N P}_{1}\right]$. inequality of income $\Rightarrow$ income's inequality.

3. $\mathbf{N P}_{\text {poss }} \Rightarrow \mathbf{N P}$. income's inequality $\Rightarrow$ income inequality.

4. $\mathbf{N P}_{\text {poss }} \Rightarrow \mathbf{N P}_{P P_{o f}}$. income's inequality $\Rightarrow$ inequality of income.

5. $\mathbf{N P}_{N C} \Rightarrow \mathbf{N P}_{\text {poss }}$. income inequality $\Rightarrow$ income's inequality.

6. $\mathbf{N P}_{N C} \Rightarrow \mathbf{N P}_{P P}$. income inequality $\Rightarrow$ inequality in incomes. 


\begin{abstract}
Sharply rising income inequality has raised the stakes of the economic game .
Sharply rising income inequality has raised the economic game 's stakes .

Sharply rising income inequality has raised the economic game stakes .

Sharply rising inequality of income has raised the stakes of the economic game .

Sharply rising inequality of income has raised the economic game 's stakes .

Sharply rising inequality of income has raised the economic game stakes .

Sharply rising inequality of incomes has raised the stakes of the economic game.

Sharply rising inequality of incomes has raised the economic game's stakes .

Sharply rising inequality of incomes has raised the economic game stakes .

Sharply rising inequality in income has raised the stakes of the economic game.

Sharply rising inequality in income has raised the economic game 's stakes.

Sharply rising inequality in income has raised the economic game stakes .

Sharply rising inequality in incomes has raised the stakes of the economic game.

Sharply rising inequality in incomes has raised the economic game's stakes.

Sharply rising inequality in incomes has raised the economic game stakes .
\end{abstract}

Table 1: Sample sentence and automatically generated paraphrases. Paraphrased NCs are in italics.

7. remove that where optional

I think that he is right $\Rightarrow$ I think he is right.

8. add that where optional

I think he is right $\Rightarrow$ I think that he is right.

where:

$\begin{array}{ll}\text { poss } & \text { possessive marker: ' or 's; } \\ \mathbf{P} & \text { preposition; } \\ \mathbf{N P}_{P P} & \text { NP with internal PP-attachment; } \\ \mathbf{N P}_{P P_{o f}} & \text { NP with internal PP headed by of; } \\ \mathbf{N P}_{\text {poss }} & \text { NP with internal possessive marker; } \\ \mathbf{N P}_{N C} & \text { NP that is a Noun Compound. }\end{array}$

While the first four and the last two transformations are purely syntactic, (5) and (6) are not. The algorithm must determine whether a possessive marker is feasible for (5) and must choose the correct preposition for (6). In either case, for noun compounds (NCs) of length 3 or more, it also needs to choose the position to modify, e.g., inquiry's committee chairman vs. inquiry committee's chairman.

In order to ensure accuracy of the paraphrases, we use statistics gathered from the Web, using a variation of the approaches presented in Lapata and Keller (2004) and Nakov and Hearst (2005). We use patterns to generate possible prepositional or copula paraphrases in the context of the preceding and the following word in the sentence, First we split the NC into two parts $N_{1}$ and $N_{2}$ in all possible ways, e.g., beef import ban lifting would be split as: (a)
$N_{1}=$ "beef", $N_{2}=$ "import ban lifting", (b) $N_{1}=$ "beef import", $N_{2}=$ "ban lifting", and (c) $N_{1}=$ "beef import ban", $N_{2}=$ "lifting". For every split, we issue exact phrase queries to the Google search engine using the following patterns:

$$
\begin{aligned}
& \text { "lt } N_{1} \text { poss } N_{2} \text { rt" } \\
& \text { "lt } N_{2} \text { prep det } N_{1}^{\prime} \text { rt" } \\
& \text { "lt } N_{2} \text { that be det } N_{1}^{\prime} \text { rt" } \\
& \text { "lt } N_{2} \text { that be prep det } N_{1}^{\prime} \text { rt" }
\end{aligned}
$$

where: $1 t$ is the word preceding $N_{1}$ in the original sentence or empty if none, $r t$ is the word following $N_{2}$ in the original sentence or empty if none, poss is a possessive marker ('s or '), that is that, which or who, be is is or are, det is a determiner (the, $a$, an, or none), prep is one of the 8 prepositions used by Lauer (1995) for semantic interpretation of NCs: about, at, for, from, in, of, on, and with, and $N_{1}^{\prime}$ can be either $N_{1}$, or $N_{1}$ with the number of its last word changed from singular/plural to plural/singular.

For all splits, we collect the number of page hits for each instantiation of each pattern, filtering out the paraphrases whose page hit count is less than 10 . We then calculate the total number of page hits $H$ for all paraphrases (for all splits and all patterns), and retain those ones whose page hits count is at least $10 \%$ of $H$. Note that this allows for multiple paraphrases of an NC. If no paraphrases are retained, we 
repeat the above procedure with lt set to the empty string. If there are still no good paraphrases, we set the $r t$ to the empty string. If this does not help either, we make a final attempt, by setting both $1 t$ and rt to the empty string.

Table 1 shows the paraphrases for a sample sentence. We can see that income inequality is paraphrased as inequality of income, inequality of incomes, inequality in income and inequality in incomes; also economic game's stakes becomes economic game stakes and stakes of the economic game.

\section{Experiments}

Table 2 shows a summary of our submissions: the official runs are marked with a $\star$. For our experiments, we used the baseline system, provided by the organizers, which we modified in different ways, as described below.

\subsection{Domain Adaptation}

All our systems were trained on both corpora.

- Language models. We used two language models (LM) - a small in-domain one (trained on News Commentary) and a big out-of-domain one (trained on Europarl). For example, for EN $\rightarrow$ ES (from English to Spanish), on the lowercased tuning data set, using in-domain LM only achieved a BLEU of 0.332910, while using both LMs yielded 0.354927, a significant effect.

- Cognates. Previous research has found that using cognates can help get better word alignments (and ultimately better MT results), especially in case of a small training set. We used the method described in (Kondrak et al., 2003) in order to extract cognates from the two data sets. We then added them as sentence pairs to the News Commentary corpus before training the word alignment models ${ }^{1}$ for $u c b 3, u c b 4$ and ucb5.

\footnotetext{
${ }^{1}$ Following (Kondrak et al., 2003), we considered words of length 4 or more, we required the length ratio to be between $\frac{7}{10}$ and $\frac{10}{7}$, and we accepted as potential cognates all pairs for which the longest common subsequence ratio (LCSR) was 0.58 or more. We repeated 3 times the cognate pairs extracted from the Europarl, and 4 times the ones from News Commentary.
}

- Phrases. The ucb5 system uses the Europarl data in order to learn an additional phrase table and an additional lexicalized re-ordering model.

\subsection{Paraphrasing the Training Set}

In two of our experiments (ucb3, ucb4 and $u c b 5)$, we used a paraphrased version of the training News Commentary data, using all rules (1)-(8). We trained two separate MT systems: one on the original corpus, and another one on the paraphrased version. We then used both resulting lexicalized re-ordering models and a merged phrase table with extra parameters: if a phrase appeared in both phrase tables, it now had 9 instead of 5 parameters (4 from each table, plus a phrase penalty), and if it was in one of the phrase tables only, the 4 missing parameters were filled with $1 \mathrm{e}-40$.

The ucb5 system is also trained on Europarl, yielding a third lexicalized re-ordering model and adding 4 new parameters to the phrase table entries.

Unfortunately, longer sentences (up to 100 tokens, rather than 40), longer phrases (up to 10 tokens, rather than 7), two LMs (rather than just one), higher-order LMs (order 7, rather than 3), multiple higher-order lexicalized re-ordering models (up to 3), etc. all contributed to increased system's complexity, and, as a result, time limitations prevented us from performing minimum-error-rate training (MERT) (Och, 2003) for $u c b 3, u c b 4$ and $u c b 5$. Therefore, we used the MERT parameter values from $u c b l$ instead, e.g. the first 4 phrase weights of $u c b 1$ were divided by two, copied twice and used in $u c b 3$ as the first 8 phrase-table parameters. The extra 4 parameters of $u c b 5$ came from training a separate MT system on the Europarl data (scaled accordingly).

\subsection{Paraphrasing the Test Set}

In some of our experiments ( $u c b 2$ and $u c b 4$ ), given a test sentence, we generated the single most-likely paraphrase, which makes it syntactically closer to Spanish and French. Unlike English, which makes extensive use of noun compounds, these languages strongly prefer connecting the nouns with a preposition (and less often turning a noun into an adjective). Therefore, we paraphrased all NCs using prepositions, by applying rules (4) and (6). In addition, we 


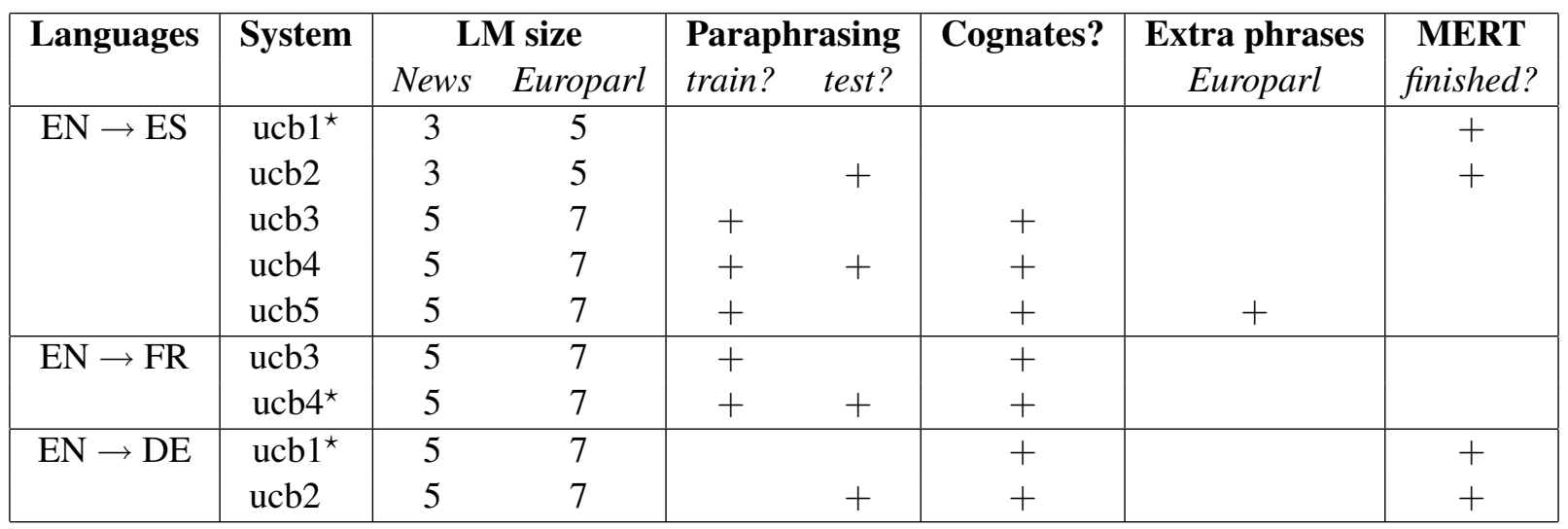

Table 2: Summary of our submissions. All runs are for the News Commentary test data. The official submissions are marked with a star.

applied rule (8), since its Spanish/French equivalent que (as well as the German $d a \beta$ ) is always obligatory. These transformations affected 927 out of the 2007 test sentences. We also used this transformed data set when translating to German (however, German uses NCs as much as English does).

\subsection{Other Non-standard Settings}

Below we discuss some non-standard settings that differ from the ones suggested by the organizers in their baseline system. First, following Birch et al. (2006), who found that higher-order LMs give better results ${ }^{2}$, we used a 5-gram LM for News Commentary, and 7-gram LM for Europarl (as opposed to 3-gram, as done normally). Second, for all runs we trained our systems on all sentences of length up to 100 (rather than 40, as suggested in the baseline system). Third, we used a maximum phrase length limit of 10 (rather than 7, as typically done). Fourth, we used both a lexicalized and distance-based reordering models (as opposed to lexicalized only, as in the baseline system). Finally, while we did not use any resources other than the ones provided by the shared task organizers, we made use of Web frequencies when paraphrasing the training corpus, as explained above.

\section{Conclusions and Future Work}

We have presented various approaches to domain adaptation and their combinations. Unfortunately,

\footnotetext{
${ }^{2}$ They used a 5-gram LM trained on Europarl, but we pushed the idea further, using a 7-gram LM with a Kneser-Ney smoothing.
}

computational complexity and time limitations prevented us from doing proper MERT for the interesting more complex systems. We plan to do a proper MERT training and to study the impact of the individual components in isolation.

Acknowledgements: This work supported in part by NSF DBI-0317510.

\section{References}

Alexandra Birch, Chris Callison-Burch, Miles Osborne, and Philipp Koehn. 2006. Constraining the phrase-based, joint probability statistical translation model. In Proc. of Workshop on Statistical Machine Translation, pages 154-157.

Dan Klein and Christopher Manning. 2003. Accurate unlexicalized parsing. In Proceedings of $A C L$ ' 03.

Philipp Koehn and Christof Monz. 2006. Manual and automatic evaluation of machine translation between european languages. In Proceedings on the Workshop on Statistical Machine Translation, pages 102-121.

Grzegorz Kondrak, Daniel Marcu, and Kevin Knight. 2003. Cognates can improve statistical translation models. In Proceedings of NAACL, pages 46-48.

Mirella Lapata and Frank Keller. 2004. The web as a baseline: Evaluating the performance of unsupervised web-based models for a range of nlp tasks. In Proceedings of HLTNAACL '04.

Mark Lauer. 1995. Corpus statistics meet the noun compound: some empirical results. In Proceedings of ACL '95.

Preslav Nakov and Marti Hearst. 2005. Search engine statistics beyond the n-gram: Application to noun compound bracketing. In Proceedings of CoNLL '05.

Franz Josef Och. 2003. Minimum error rate training in statistical machine translation. In Proceedings of $A C L$, pages 160-167. 